Check for updates

Cite this: Chem. Commun., 2022, 58,1756

Received 13th August 2021

Accepted 4th January 2022

DOI: $10.1039 / \mathrm{d} 1 \mathrm{cc} 04455 a$

rsc.li/chemcomm

\section{Depth prediction of nanotags in tissue using surface enhanced spatially offset Raman scattering (SESORS) †+}

\author{
Matthew E. Berry, (D) a Samantha M. McCabe, (D) a Neil C. Shand, (D) ${ }^{b}$ \\ Duncan Graham (D) and Karen Faulds (D) *a
}

\begin{abstract}
A model for the prediction of the depth of two 'flavours' of surface enhanced Raman scattering (SERS) active nanotags embedded within porcine tissue is demonstrated using ratiometric analysis. Using a handheld spatially offset Raman (SORS) instrument, SESORS signals could be detected from nanotags at depths down to $48 \mathrm{~mm}$ for the first time using a backscattering SORS geometry.
\end{abstract}

In recent years, surface enhanced Raman scattering (SERS) has been used extensively in bioanalytical research applications with the goal of creating platforms for medical diagnostics. ${ }^{1,2}$ SERS is a powerful analytical technique that can be used to detect target molecules in close proximity to the surface of a metal nanostructure, which upon interrogation with a monochromatic laser will provide enhancements through a localised electromagnetic field. ${ }^{3,4}$ Theoretical electromagnetic enhancement factors of $10^{11}$ have been reported, vastly improving detection sensitivity over Raman spectroscopy alone..$^{5}$ In addition to favourable signal enhancements, the use of nanoparticles (NPs) with an amenable surface chemistry, such as gold and silver, means that it is possible to develop specific biofunctional nanotags that can be optimised for in vivo bioanalytical detection by coupling NPs with Raman reporters (molecules with a large Raman cross section) and targeting biomolecules to interact with biomarkers of interest. ${ }^{6}$ Despite the numerous advantages offered by SERS, non-invasive subsurface detection remains a challenge as most Raman systems are limited by surface selectivity. This is a drawback that has hindered the transition of the technique into in vivo diagnostic applications.

\footnotetext{
${ }^{a}$ Centre for Molecular Nanometrology, Department of Pure and Applied Chemistry, University of Strathclyde, 99 George Street, Glasgow, G1 1RD, UK.

E-mail: karen.faulds@strath.ac.uk

${ }^{b}$ Defence Science and Technology Laboratory (DSTL), Porton Down, Salisbury, SP4 oJQ, UK

$\dagger$ Research data associated with this paper will become available through the following link: https://doi.org/10.15129/057607c4-fe19-4546-8ef12bf5ed5742d. \# Electronic supplementary information (ESI) available. See DOI: 10.1039/ d1cc04455a
}

Spatially offset Raman spectroscopy (SORS) is a technique that allows for the non-invasive retrieval of subsurface Raman spectra from within diffusely scattering materials such as plastic, glass and mammalian tissue. ${ }^{7-10}$ In contrast to conventional Raman, SORS rejects surface photons through the use of excitation and collection regions separated by a spatial offset $(\mathrm{d} x) .{ }^{11}$ By altering $\mathrm{d} x$, the spectral contribution of the surface layer of a diffusely scattering sample diminishes and the signals from deeper layers dominate a typical SORS spectrum.

The field of surface enhanced, spatially offset Raman spectroscopy (SESORS) has emerged to combine the signal enhancements offered by SERS with the subsurface probing of diffusely scattering media offered by SORS, with the aim of performing measurements in vivo at depths superior to those performed on conventional Raman instruments. ${ }^{12-14}$ SESORS has shown promise in the field of medical diagnostics. The technique has been demonstrated to be effective in the through tissue tracking of osteoporosis drugs on bone, the multiplexed detection of neurotransmitters through bone, the multiplexed imaging of live tumour models through tissue using a handheld device, and the in vivo detection and imaging of glioblastoma in mice. ${ }^{15-19}$ It is clear that deep Raman techniques such as SESORS have the potential to be used as diagnostic tools with a wide range of clinical applications in the future. ${ }^{20}$

In this work we report a proof-of-concept approach for the prediction of the depth of SERS active nanotags embedded within up to $48 \mathrm{~mm}$ worth of porcine tissue using ratiometric analysis of the Raman intensities of the nanotags and the tissue barrier, or the "relative contribution" of the nanotags to through tissue spectra. Other calibration techniques have been reported that exploit the differential attenuation of Raman intensities within different wavenumber regions in a spectrum brought about by the absorption properties of the surface barrier. ${ }^{21,22}$ Our approach differs from others in that it exploits the exponential decay in the relative contribution of the SERS active nanotags in SORS spectra. As the tissue barrier thickness is increased incrementally, a linear calibration is created that 
correlates the Raman response of the multi-layered system with the depth of the buried SERS active inclusion. We have evaluated this approach using a handheld backscattering SORS spectrometer in contact with a porcine tissue model and two different 'flavours' of SERS active nanotags, with the main objective being the development of a calibration model that can be used with known concentrations of nanotags that can be applied to a wide range of Raman reporters, bringing SESORS closer to a point-of-use application in a clinical setting. ${ }^{23}$

To develop SERS active nanotags that produce an optical response when buried within tissue at depth, silica coated gold NP (AuNP) aggregates were synthesised according to previously reported methods. ${ }^{24}$ Briefly, AuNPs with an average size of $50 \mathrm{~nm}$ were subjected to controlled aggregation by mixing with two commercially available Raman reporters, 1,2-bis(4pyridyl)ethylene (BPE) and 4-(1H-pyrazol-4-yl)pyridine (PPY), and then encapsulation with a silica shell to give two NP flavours with unique, high intensity SERS fingerprints when interrogated with a near infrared (NIR) laser, as shown in Fig. S1 (ESI $\ddagger$ ). Aggregation was monitored by observing colour changes in the colloidal suspensions and simultaneously recording the SERS response of aliquots taken from the reaction mixtures. The nanotags were characterised by extinction spectroscopy, dynamic light scattering (DLS), and zeta potential analysis and these results can be observed in Fig. S2 (ESI ). DLS analysis indicated that the nanotags had increased in size after aggregation and encapsulation to 109 and $71 \mathrm{~nm}$ for PPY and BPE, respectively. Additionally, zeta potential analysis indicated that the nanotag samples were negatively charged, with zeta potential values of -36 and $-44 \mathrm{mV}$ reported for PPY and BPE, and hence stable in suspension. The nanotag samples feature a localised surface plasmon resonance (LSPR) at 535 and $530 \mathrm{~nm}$ and low intensity extinction bands from aggregated NPs were observed at 770 and $740 \mathrm{~nm}$ for PPY and BPE, respectively. These extinction bands in the NIR spectral window mean that the nanotags are optically active when probed using the $830 \mathrm{~nm}$ laser excitation wavelength used in this study and produce larger Raman signals compared to bare AuNP conjugates. ${ }^{25}$ The experimental setup for SESORS measurements is described in Fig. S3 (ESI $\$)$. Nanotag solutions $\left(350 \mu \mathrm{L}, 248 \mathrm{pM}\left(5.4 \times 10^{10}\right.\right.$ particles $)$ PPY; $782 \mathrm{pM}\left(1.6 \times 10^{11}\right.$ particles $\left.) \mathrm{BPE}\right)$ were pipetted into a quartz microcuvette, path length $5 \mathrm{~mm}$, and the cuvette then transferred to a section of lean porcine back tissue. Another section of porcine tissue with a thickness of $3 \mathrm{~mm}$ was placed on top of the nanotags. A translational $z$-stage was used to manoeuvre the samples and for each measurement they were raised vertically until they were in contact with the nose cone of a handheld SORS spectrometer and resistance was experienced when turning the stage handle. The tissue barrier between the laser and the nanotags was increased to 20 layers $(60 \mathrm{~mm})$ incrementally.

Using spectra that were collected at an $8 \mathrm{~mm}$ offset and subsequently averaged, baselined, and normalised, SESORS stack plots containing through barrier measurements and references for the nanotag and tissue for both Raman reporters were constructed, Fig. 1A and B. The contribution of the PPY and BPE nanotags to the spectra was determined by tracking
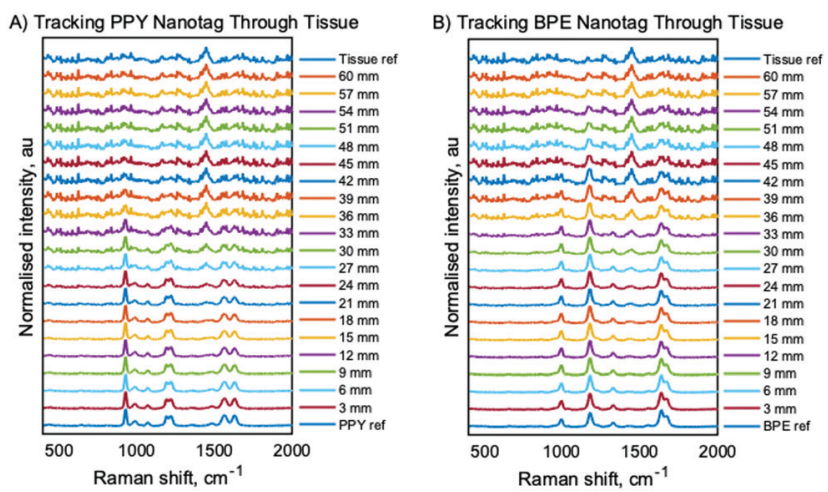

Fig. 1 The tracking of (A) PPY and (B) BPE nanotags through porcine tissue up to $60 \mathrm{~mm}$ using a handheld SORS spectrometer. The spectra at the bottom of the plots show the reference spectra from the nanotags in the absence of a barrier and the spectra at the top refer to reference spectra from the porcine tissue, respectively. SESORS spectra were recorded in triplicate at each depth and for each reference and were subsequently averaged, stacked, and normalised for clarity. Measurements were carried out using a 2 second integration time, 6 accumulations and an $830 \mathrm{~nm}$ laser excitation wavelength.

the bands at $928 \mathrm{~cm}^{-1}$ and $1178 \mathrm{~cm}^{-1}$ and the tissue barrier contribution to the spectra was determined by tracking the $1444 \mathrm{~cm}^{-1}$ band. The two SERS bands were selected over Raman shift bands at higher wavenumbers because they lie in a spectral window with low water absorption. Bands above $1500 \mathrm{~cm}^{-1}$ are subject to a different rate of exponential decrease through tissue due to a larger water absorption profile. ${ }^{21}$ It can be observed from the plots that the contribution of the nanotag in the SESORS spectra decreases with increasing depth until the tissue barrier dominates at large tissue barriers for both Raman reporters. The PPY nanotags were detected at $42 \mathrm{~mm}$ (14 layers) and the BPE nanotags could be detected at $48 \mathrm{~mm}$ (16 layers). The depths of detection reported in this work are, to the best of our knowledge, the highest buried depths at which SERS active nanotags have been detected using a backscattering SORS system. Principal component analysis (PCA) was applied to analyse the data from the SESORS stack plots to establish the Raman bands with the highest variance across the depth measurement spectra and the spectra corresponding to the NP and tissue barrier reference spectra, Fig. S4 (ESI $\ddagger$ ). ${ }^{26}$ The PCA loadings were plotted against the Raman shift and indicate that, for both Raman reporters there is a distinct separation between spectra with a high NP contribution buried at lower depths compared to spectra with a high tissue contribution at larger depths. The PCA loadings also support the selection of the Raman bands for the through tissue tracking of the nanotags because the bands used in the study represent the largest source of variance across all spectra recorded in the respective experiments in the first and second principal components (PCs). At depths where the nanotag SERS signatures dominate through tissue spectra, the spectra can be discriminated primarily in PC1, but at depths where the tissue Raman signatures dominate spectral discrimination comes primarily from PC2. For both nanotags, the PC2 loading causes spectra to cluster negatively in the absence of either one of the tissue Raman or nanotag SERS signatures, and it follows that at 
large tissue barriers, the negative clustering of spectra indicates insufficient contribution of the nanotags. Therefore, we have determined that the maximum depth penetration of the nanotags corresponds to the largest depth that gives rise to positive clustering in PC2.

The focus of this work is to demonstrate the ability of ratiometric analysis of the nanotags versus Raman tissue signatures to determine the depth location of the nanotags behind the tissue barrier and to develop calibration models for both nanotag flavours. To achieve this, offset spectra were acquired at the point of maximum nanotag intensity through a tissue barrier with the thickness increasing in $3 \mathrm{~mm}$ steps until the nanotag SERS signatures were no longer visually distinguishable and the spectra no longer clustered positively in PC2 in the PCA analysis, Fig. S4 (ESI ). This occurred at depths of $42 \mathrm{~mm}$ for PPY and $48 \mathrm{~mm}$ for BPE. An offset of $8 \mathrm{~mm}$ was applied for all measurements which is the largest available on the handheld SORS spectrometer used. Spectra were measured in triplicate at a given depth and were subsequently averaged and baselined before the Raman intensity of the tissue band at $1444 \mathrm{~cm}^{-1}$ and the nanotag bands at $928 \mathrm{~cm}^{-1}$ for PPY and $1178 \mathrm{~cm}^{-1}$ for BPE were recorded. The natural logarithm of the ratio of the NP and tissue Raman intensities $\left(928 \mathrm{~cm}^{-1} / 1444 \mathrm{~cm}^{-1}\right.$ for PPY and $1178 \mathrm{~cm}^{-1} / 1444 \mathrm{~cm}^{-1}$ for BPE) was plotted against the depth at which the nanotags were buried, Fig. 2, and linear calibration plots were obtained with coefficients of determination $\left(R^{2}\right)$ of $98.92 \%$ and $99.24 \%$ for PPY and BPE, respectively. It is worth noting that the calibration curves for the different nanotags have similar gradients, indicating that the decay in the intensity ratio is related to the optical properties of the tissue and is independent of the SERS intensity of the nanotag. The use of the natural logarithm of the intensity ratio was justified by comparing the regressions obtained for both nanotags against those developed with the natural logarithm applied to the depth, Fig. S5 (ESI ). It was observed that when the logarithm was applied to the intensity ratio, the variables were more highly correlated and the datasets had less skewed distributions, meaning that the chosen method was more suitable for calibration. Inset plots of the ratio of the PPY and BPE nanotags and tissue Raman intensities against the buried depth are shown and this illustrates the exponential decay that occurs in the intensity ratio as the thickness of the tissue barrier is increased between the nanotags and the laser. In the BPE inset, the ratio at $6 \mathrm{~mm}$ is slightly larger than at $3 \mathrm{~mm}$, and this was attributed to variations in the pressure applied to the tissue barrier by the spectrometer nose cone. This result was used in the development of the calibration models depicted in Fig. $2 \mathrm{~A}$ and B.

To investigate the ability of the calibration technique to establish the depth of the nanotags based on log-linear regression of the intensity ratio $I_{\mathrm{NP}} / I_{\text {Tis }}$, prediction studies were undertaken. The nanotags were buried at various measured depths in samples calibrated over the previously identified maximum depth penetrations and SESORS measurements were performed by a researcher with no prior knowledge of the depth location of the nanotags. The models were then used to predict the depth of the nanotags based upon the SERS response of the

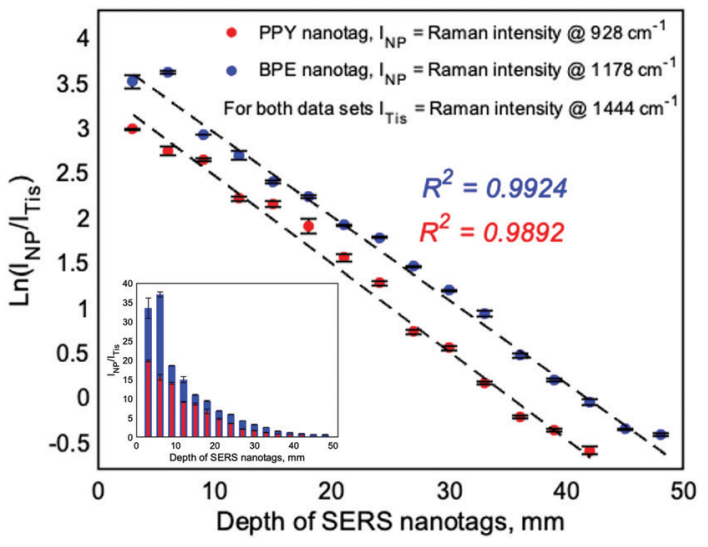

Fig. 2 Calibration plots correlating the Raman response of the multi-layered tissue and nanotag samples and the depth of the nanotags buried within the tissue. The data in red shows the plot of the natural logarithm of the averaged intensity ratio $928 \mathrm{~cm}^{-1} / 1444 \mathrm{~cm}^{-1}\left(\mathrm{Ln}\left(/_{\mathrm{NP}} / /_{\text {Tis }}\right)\right)$ against depth of the SERS active PPY nanotag that shows a strong linear relationship between $3 \mathrm{~mm}$ and $42 \mathrm{~mm}$ with a coefficient of determination of $98.92 \%$. The data in blue shows the plot of the natural logarithm of the averaged intensity ratio $1178 \mathrm{~cm}^{-1}$ / $1444 \mathrm{~cm}^{-1}\left(\mathrm{Ln}\left(/_{\mathrm{NP}} / I_{\mathrm{TiS}}\right)\right)$ against depth of the SERS active BPE nanotag that shows a strong linear relationship between $3 \mathrm{~mm}$ and $48 \mathrm{~mm}$ with a coefficient of determination of $99.24 \%$. The inset shows plots of the intensity ratio $I_{\mathrm{NP}} / I_{\text {Tis }}$ against the depth of SERS active PPY (red) and BPE (blue) nanotags. The error bars in each plot represent \pm one standard deviation from three recorded scans at a specific depth.

samples. The predicted depths were plotted against the measured depths and the root-mean-square error of calibration (RMSEC), a measure of the differences between values predicted by the model and values observed, were calculated as $1.956 \mathrm{~mm}$ and $2.127 \mathrm{~mm}$ for the PPY and BPE calibrations, respectively, Fig. 3. The error values reported correspond to percentage errors of $5.015 \%$ and $4.727 \%$ of the $39 \mathrm{~mm}$ and $45 \mathrm{~mm}$ depth ranges over which the calibrations were developed. Both calibration models allowed the depth of the buried nanotags to be accurately predicted and the corresponding errors are the lowest reported values for the prediction of SERS active nanotag depth through tissue using a backscattering SORS configuration, with alternative approaches citing a percentage error of $6.5 \% .^{21}$

Using an internal calibration technique based upon ratiometric analysis of the Raman intensities of the subsurface nanotags and the surface tissue barrier obtained in SESORS measurements has allowed prediction of the depth of two distinct NP flavours through tissue using a handheld SORS spectrometer. Two nanotags were used to demonstrate that this approach can be used equally on nanotags with different SERS intensities and depth penetration capabilities. The experiments reported here used fixed amounts of NPs and this would not be the case in a real clinical sample which is the target of our next sets of experiments, i.e., to link the signal at depth to concentration of NPs present. This will be challenging but we believe it is possible by using well calibrated batches of NPs with highly uniform signal intensities. The end goal of SESORS research is the non-invasive in vivo detection of disease in humans, and so it follows that it will be crucial to apply this technique within 


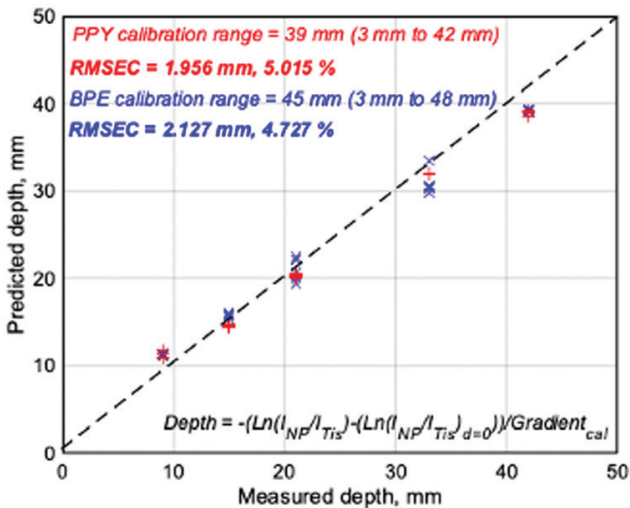

Fig. 3 Depth prediction plots for the PPY and BPE nanotags. The data in red shows the predicted against measured depths plotted for the PPY nanotags. Five predictive measurements were recorded at each depth, and a root-mean-square error of calibration (RMSEC) of $1.956 \mathrm{~mm}$ or $5.015 \%$ of the calibration range, was observed. The data in blue shows the predicted against measured depths plotted for the BPE nanotags. Five predictive measurements were recorded at each depth and a RMSEC of $2.127 \mathrm{~mm}$, or $4.727 \%$ of the calibration range, was observed. The inset equation describes the relationship between the predicted depth, the natural logarithm of the intensity ratio of the NP and tissue Raman intensities from the previously developed linear calibrations. For RMSEC calculations, a sample size of $\mathrm{N}-2$ was used to reduce sample bias.

biological models using nanotags that have targeting capabilities and are biocompatible in terms of their size and surface modifications. Additional future work will involve the development of external depth calibration curves, calibrations on optically heterogeneous tissue samples, comprising of organs, muscle, fat, and skin, the calibration of multiple nanotags simultaneously in a single sample, and the application of the calibration technique to SESORS imaging to test samples of different sizes, investigate prediction of their position within 3D space, and understanding the effect that changes in concentration across a sample will have on the calibrations. To the best of our knowledge the through tissue detection depths reported here are the largest achieved using a backscattering configuration with calibrations possible at tissue thicknesses up to and including $48 \mathrm{~mm}$ using a combination of nanotag aggregates and pressure applied from the nose cone of the spectrometer on the samples. Furthermore, the calibrations were found to be highly accurate with lower error values (5.015 and $4.727 \%$ RMSEC for the respective NP flavours) than previously reported calibration techniques using a backscattering SORS system. ${ }^{21}$ The results described here further support the excellent potential that user friendly handheld SORS spectrometers have in clinical applications since quantification and imaging of SERS active nanotags through tissue have previously been reported using the same system. ${ }^{17,27}$ The ability to determine the depth of SERS nanotags through tissue in in vivo applications is a significant challenge, however through the use of a novel calibration technique coupled with a handheld SORS system this work represents a significant step forward in the ability to determine the depth of multiple vibrational fingerprints pertaining to SERS nanotags in a clinical setting.
MEB acknowledges the help of Rachel McGee at Agilent Technologies. This work was supported by the Engineering and Physical Sciences Research Council (EPSRC) and Medical Research Council (MRC) through the CDT in Optical Medical Imaging (OPTIMA), grant number EP/L016559/1 for funding MEB, DG and KF, and DSTL for funding SMM, DG and KF.

\section{Conflicts of interest}

There are no conflicts to declare.

\section{References}

1 S. Laing, K. Gracie and K. Faulds, Chem. Soc. Rev., 2016, 45, 1901-1918.

2 M. E. Berry, H. Kearns, D. Graham and K. Faulds, Analyst, 2021, 146, 6084.

3 D. L. Jeanmaire and R. P. V. Duyne, J. Electroanal. Chem., 1977, 84, $1-20$.

4 K. A. Willets and R. P. Van Duyne, Annu. Rev. Phys. Chem., 2007, 58, 267-297.

5 E. Hao and G. C. Schatz, J. Chem. Phys., 2003, 120, 357-366.

6 S. Laing, L. E. Jamieson, K. Faulds and D. Graham, Nat. Rev. Chem., 2017, 1, 0060.

7 P. Matousek, I. P. Clark, E. R. C. Draper, M. D. Morris, A. E. Goodship, N. Everall, M. Towrie, W. F. Finney and A. W. Parker, Appl. Spectrosc., 2005, 59, 393-400.

8 P. Matousek, M. D. Morris, N. Everall, I. P. Clark, M. Towrie, E. Draper, A. Goodship and A. W. Parker, Appl. Spectrosc., 2005, 59, 1485-1492.

9 P. Matousek, Chem. Soc. Rev., 2007, 36, 1292-1304.

10 P. Matousek, Appl. Spectrosc., 2006, 60, 1341-1347.

11 S. Mosca, C. Conti, N. Stone and P. Matousek, Nat. Rev. Dis. Primers, 2021, 1, 1-16.

12 N. Stone, M. Kerssens, G. R. Lloyd, K. Faulds, D. Graham and P. Matousek, Chem. Sci., 2011, 2, 776-780.

13 N. Stone, K. Faulds, D. Graham and P. Matousek, Anal. Chem., 2010, 82, 3969-3973.

14 S. M. Asiala, N. C. Shand, K. Faulds and D. Graham, ACS Appl. Mater. Interfaces, 2017, 9, 25488-25494.

15 H. Xie, R. Stevenson, N. Stone, A. Hernandez-Santana, K. Faulds and D. Graham, Angew. Chem., Int. Ed., 2012, 51, 8509-8511.

16 A. S. Moody, P. C. Baghernejad, K. R. Webb and B. Sharma, Anal. Chem., 2017, 89, 5688-5692.

17 F. Nicolson, L. E. Jamieson, S. Mabbott, K. Plakas, N. C. Shand, M. R. Detty, D. Graham and K. Faulds, Chem. Sci., 2018, 9, 3788-3792.

18 F. Nicolson, L. E. Jamieson, S. Mabbott, K. Plakas, N. C. Shand, M. R. Detty, D. Graham and K. Faulds, Chem. Commun., 2018, 54, $8530-8533$.

19 F. Nicolson, B. Andreiuk, C. Andreou, H.-T. Hsu, S. Rudder and M. F. Kircher, Theranostics, 2019, 9, 5899-5913.

20 B. Gardner, P. Matousek and N. Stone, Analyst, 2019, 144, 3552-3555.

21 S. Mosca, P. Dey, T. A. Tabish, F. Palombo, N. Stone and P. Matousek, J. Biophotonics, 2019, e201960092.

22 S. Mosca, P. Dey, T. A. Tabish, F. Palombo, N. Stone and P. Matousek, Anal. Chem., 2019, 91, 8994-9000.

23 F. Nicolson, L. E. Jamieson, S. Mabbott, N. C. Shand, D. Graham and K. Faulds, J. Raman Spectrosc., 2017, 48, 1828-1838.

24 J. F. Li, X. D. Tian, S. B. Li, J. R. Anema, Z. L. Yang, Y. Ding, Y. F. Wu, Y. M. Zeng, Q. Z. Chen, B. Ren, Z. L. Wang and Z. Q. Tian, Nat. Protoc., 2013, 8, 52-65.

25 H. Kearns, N. C. Shand, W. E. Smith, K. Faulds and D. Graham, Phys. Chem. Chem. Phys., 2015, 17, 1980-1986.

26 H. Kearns, R. Goodacre, L. E. Jamieson, D. Graham and K. Faulds, Anal. Chem., 2017, 89, 12666-12673.

27 F. Nicolson, L. E. Jamieson, S. Mabbott, K. Plakas, N. C. Shand, M. R. Detty, D. Graham and K. Faulds, Analyst, 2018, 143, $5358-5363$. 